Revue d'histoire de l'Amérique française

REVUE D.HISTOIRE DE L'AMÉRIQUE FRANÇAISE

\title{
Le village en étoile, innovation des Jésuites et non de Talon
}

\section{Marcel Trudel}

Volume 44, numéro 3, hiver 1991

URI : https://id.erudit.org/iderudit/304900ar

DOI : https://doi.org/10.7202/304900ar

Aller au sommaire du numéro

Éditeur(s)

Institut d'histoire de l'Amérique française

ISSN

0035-2357 (imprimé)

1492-1383 (numérique)

Découvrir la revue

Citer cette note

Trudel, M. (1991). Le village en étoile, innovation des Jésuites et non de Talon. Revue d'histoire de l'Amérique française, 44(3), 397-406.

https://doi.org/10.7202/304900ar d'utilisation que vous pouvez consulter en ligne.

https://apropos.erudit.org/fr/usagers/politique-dutilisation/ 
NOTES DE RECHERCHE

\title{
LE VILLAGE EN ÉTOILE, INNOVATION DES JÉSUITES ET NON DE TALON ${ }^{1}$
}

\author{
MARCEL TRUDEL
}

Du temps des Cent-Associés, le peuplement rural s'était conformé à une disposition particulière: des habitations situées en une seule et même ligne, sur le front de chaque parcelle de terre, celle-ci concédée en forme de rectangle étroit et allongé. Disposition que l'on qualifiera de dispersion, les habitants (écrit-on en 1665) s'étant établis «où il leur a plû, et sans se précautionner de joindre [leurs maisons] les unes aux autres, et faire leurs defrichemens de proche en proche pour mieux s'entre secourir au besoin ${ }^{2}$. Jugement que nuancera le gouverneur Buade de Frontenac: «je ne crois pas que les habitations soient trop éloignées les unes des autres, puisque les maisons n'y sont que de quatre en quatre arpents.» ${ }^{3}$ Dès 1663 , les autorités avaient souhaité modifier cette méthode de peuplement, en réduisant «les habitations en la forme de nos paroisses et de nos bourgs, autant qu'il serait dans la possibilité» ${ }^{4}$.

Des villages ou bourgs, il y en avait déjà une dizaine et qui dataient des Cent-Associés. Si certains ne sont toujours qu'à l'état embryonnaire, d'autres sont bien structurés, comme c'est le cas de Fargy dans la seigneurie de Beauport, du fort Saint-François-Xavier dans Sillery ou du fort Saint-François dans le Cap-de-la-Madeleine, sans compter Montréal et les Trois-Rivières que l'on peut alors assimiler à des bourgs. Toutefois, les colons qui y avaient leurs habitations, demeuraient isolés de leurs terres ${ }^{5}$. Or on cherchait une formule

1 Les commentaires que j'ai reçus du Comité de rédaction m'ont été fort utiles. Je tiens aussi à remercier, d'une façon particulière, Michel Gaumond, archéologue et historien, qui a bien voulu me communiquer, avant que cet article aille sous presse, les résultats d'une recherche qu'il mène depuis déjà quelques années sur ce même sujet, résultats qu'il n'a pas encore publiés.

2 Instructions à Talon, 27 mars 1665, RAPQ (1930-31): 8.

3 Buade de Frontenac à Colbert, 2 novembre 1672, RAPQ (1926-27): 13.

4 Rappelé dans Instructions ci-haut citées, note 2.

5 Sur ces villages, voir nos Débuts du régime seigneurial, 105-115. 
qui permît de regrouper les colons en un «corps de communauté», tout en laissant chacun vivre sur son lot. La solution viendra des Jésuites.

Après avoir couvert de concessions le front de leur seigneurie Notre-Dame-des-Anges (de la rivière de Beauport au confluent des rivières Saint-Charles et Lairet), les Jésuites forment en février 1665 un nouveau noyau de peuplement, à environ 5 kilomètres à l'intérieur de cette seigneurie, noyau qui reçoit le nom de Charlesbourg.

Or, en examinant attentivement le greffe du notaire Paul Vachon pour les actes de février 1665, j'ai trouvé des actes de concession qui m'ont étonné, parce qu'ils révèlent que les Jésuites mettent en application un tout nouveau plan d'aménagement.

Quel est ce plan? Les Jésuites délimitent d'abord une réserve, dite trait-quarré, de forme rectangulaire, de 5 arpents de côté: soit d'une superficie de 25 arpents, dont 5 serviront à leurs bâtiments et les 20 autres pourront tenir lieu de commune. Sur chaque côté de ce traitquarré, viennent s'abouter, par un front d'un demi-arpent ${ }^{6}$, des terres de 40 arpents de superficie: à mesure qu'on s'éloigne du front (sur lequel est construite l'habitation du censitaire), chaque terre va s'élargissant, selon un angle dûment calculé des frontières latérales, pour atteindre en leur extrémité une largeur de 4 arpents. Chacun des côtés du trait-quarré servant de front à 10 terres, on a ainsi, en principe, un total de 40 terres qui, à cause de leur tracé en figure de trapèze, forme une grande étoile.

La concession écrite de ces terres de 40 arpents chacune se fait du 22 au 28 février 1665, c'est-à-dire (ici le rappel est important) un mois avant la nomination de Talon au poste d'intendant de la NouvelleFrance et six mois avant qu'il n'arrive à Québec pour prendre sa charge. L'acte de concession, qui fait suite à une occupation plus ou moins longue des lieux ${ }^{7}$, précise chaque fois la largeur du front, celle de la profondeur à l'extrémité de la terre, la longueur de chacun des côtés et l'angle que forment ces côtés sur le trait-quarré.

Pour bien observer cette méthode, nous donnons ici le contenu de deux actes de concession ${ }^{8}$, celui de la terre de Michel Chrétien (elle est sur le côté qui s'oriente sud-ouest quart d'ouest et une de ses limites latérales est à angle droit avec le trait-quarré) et celui de la terre de Jacques Bédard, son voisin en amont:

6 L'arpent mesure 10 perches ou 180 pieds français ou 0,059 kilomètre; la perche: 18 pieds français ou 5,9 mètres.

7 Des actes mentionnent des voisins qui seraient déjà sur les lieux, bien qu'ils ne reçoivent leur titre que plus tard.

8 Ces deux actes de concession, l'un du 23 février, l'autre du 27 suivant, ainsi que les 28 autres du 22 au 28 février 1665 qui servent à construire ce village en étoile, sont tirés du greffe de Paul Vachon, aux Archives nationales. 
FIGURE 1

\section{Le village-étoile de Charlesbourg (partie frontale seulement) \\ par Marcel Trudel}

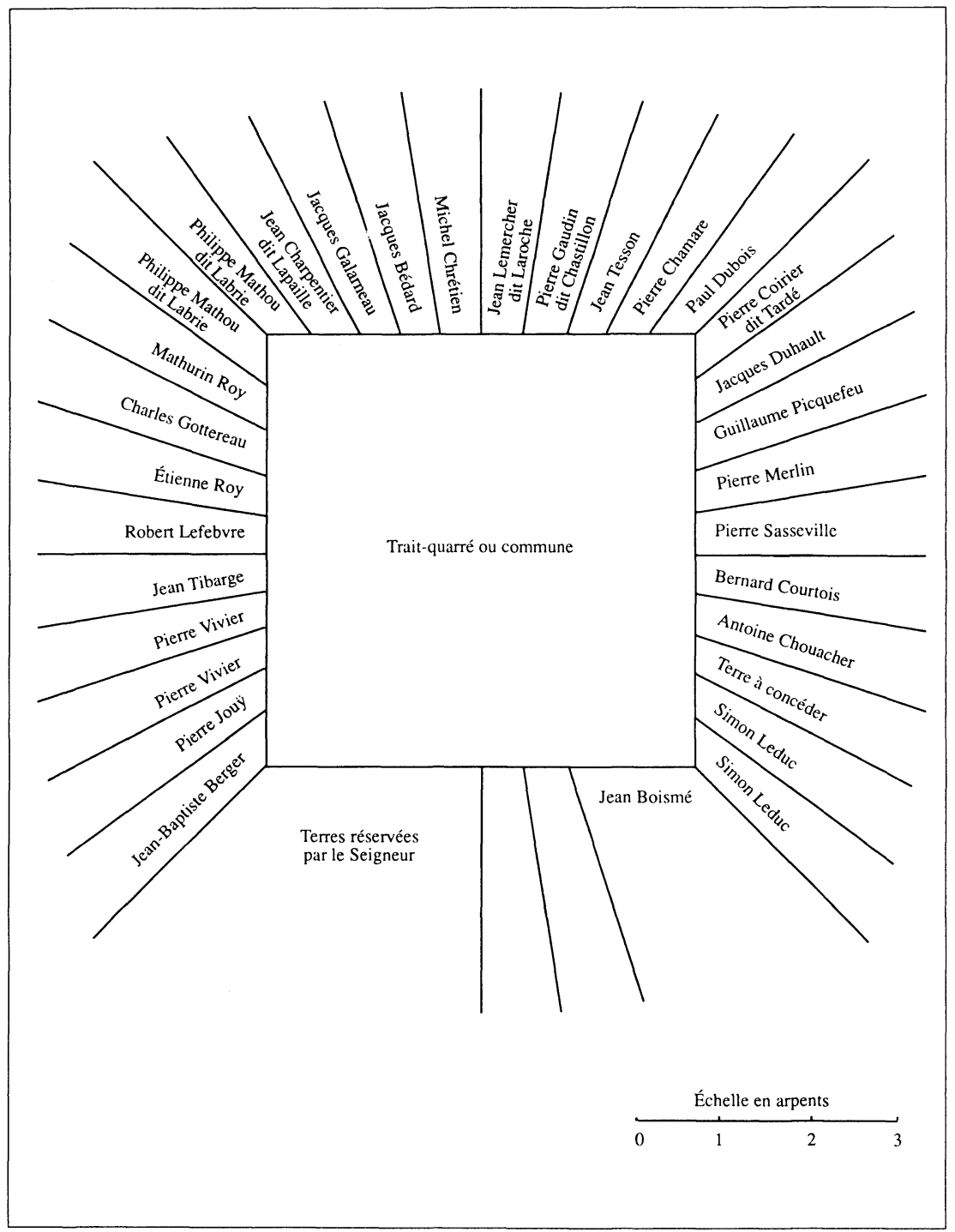

Source: Actes de concessions, 22-28 février 1665: ANQ, greffe du not. Paul Vachon. 
Michel Chrétien, concession de 40 arpents, 27 février $1665^{9}$.

front: largeur d'un demi-arpent, orienté sud-ouest quart d'ouest profondeur: largeur de 4 arpents, parallèle au front

limite latérale qui le sépare de son voisin Lemercher en aval: ligne de 17 arpents 6 perches 10 pieds, formant un angle droit avec le trait-quarré

limite latérale qui le sépare de Bédard en amont: ligne de 17 arpents 9 perches 10 pieds, formant un angle de $11^{\circ} 25^{\prime}$ vers l'ouest quart de nord-ouest

Jacques Bédard, concession de 40 arpents, 23 février 1665.

front: largeur d'un demi-arpent, orienté sud-ouest quart d'ouest profondeur: largeur de 4 arpents, parallèle au front

limite latérale qui le sépare de Chrétien en aval: ligne de 17 arpents 9 perches 10 pieds, formant un angle de $11^{\circ} 25^{\prime}$ vers l'ouest quart de nord-ouest

limite latérale qui le sépare de Galerneau en amont: ligne de 18 arpents 5 perches 1 pied, formant un angle de $21^{\circ} 47^{\prime}$ vers l'ouest quart de nord-ouest.

Et ainsi de suite dans les actes de concession des autres terres qui aboutent au trait-quarré.

Ainsi donc, en février 1665, les Jésuites ont appliqué dans leur fief Notre-Dame-des-Anges une division des terres jusque-là inconnue en ce pays. Au lieu d'un rang rectiligne où les habitants sont éloignés les uns des autres et au lieu d'un village où ils seraient regroupés à l'écart de leurs terres, voici, imaginé par les Jésuites, un habitat en forme d'étoile: chaque habitant, titulaire d'un lot de quarante arpents en forme de trapèze (et non de triangle, comme on l'écrit souvent), demeure sur sa terre, à seulement une trentaine de mètres de ses voisins, tout en faisant partie du village.

Exécuté en ce février 1665, ce plan en étoile (que nous reproduisons sur la figure 1) prévoit 40 terres, soit 10 de chaque côté du trait-quarré. En fait, il en compte déjà 28 de 40 arpents chacune, plus une terre, celle de Boismé, qui ne suit pas rigoureusement ce plan ${ }^{10}$. L'opération établit ainsi 28 censitaires (certains ont deux terres), chacun sur sa terre et tous ensemble dans un bourg carré. Réalisation toute nouvelle que peut-être apercevait-on du Cap-aux-Diamants, mais dont en tout cas l'originalité devait alors faire sensation.

9 Selon le plan de Catalogne (reproduit dans Trudel, Atlas de la Nouvelle-France, 166), un Chrétien occupe encore cette terre en 1709.

10 À l'illustration que nous avons distribuée à nos auditeurs, lors du congrès d'octobre 1990, nous apportons ici 3 corrections qui nous ont été suggérées par Michel Gaumond: la terre de Berger doit suivre le même dessin que celle de Jouy; la terre dite de Chauvin ne sera concédée qu'en 1666; la terre dite «à concéder», entre Boismé et Leduc, l'a été à ce même Leduc. 
Quel est le mathématicien qui est à l'origine de cette savante géométrie? Un jésuite du Collège de Québec? Le donné Martin Boutet, alors professeur de mathématiques en ce même Collège? Ou bien l'arpenteur Jean Bourdon, qui avait depuis trente ans la pratique de l'arpentage?

Tout à fait inconnue au pays, cette formule du village en étoile était-elle l'imitation d'un modèle rencontré ailleurs? Y avait-il de ces villages en France ou en Europe? Pour sa part, l'archéologue et historien Michel Gaumond a retrouvé, sur une carte de la Pennsylvanie, datée des environs de 1687, deux villages voisins l'un de l'autre, à 35 kilomètres au nord-est de Philadelphie, qui ont presque exactement ce plan de Charlesbourg: au centre, un carré auquel viennent s'abouter des terres en forme de trapèze ${ }^{11}$.

En tout cas, voici qu'à la fin du mois de mars 1665, un nouvel intendant, Jean Talon, reçoit à Paris des instructions royales pour corriger la dispersion qu'on reproche aux Canadiens de la campagne: il devra voir à ce que désormais les établissements ruraux se fassent de proche en proche et qu'on réduise «les habitations en la forme de nos paroisses et de nos bourgs, autant qu'il serait dans la possibilité» ${ }^{12}$.

Talon débarque à Québec six mois plus tard. Empressé de bien servir, il s'applique tout de suite à chercher le plan qui réponde à cette politique et il écrit au ministre Colbert: «Je projette une forme de desfrichement pour bastir une première bourgade; quand elle sera tout à fait résolue, je vous en envoyerai le plan..$^{13}$ Quelle est cette forme qu'il projette: celle qu'il a sous les yeux et dont il ne peut pas ne pas avoir entendu parler? En novembre 1666, il poursuit son travail: «Je travaille autant que je puis, écrit-il à Colbert, à reunir et raprocher Les habitations esloignées et je m'oppose à ce qu'à l'avenir on en forme aucune qui ne soit en corps de communauté, hameau, Vilages ou Bourgs.» ${ }^{14}$ En janvier de l'année suivante, les autorités de Québec émettent un règlement qui sanctionne ce projet de «former des villages en corps de communauté». On reconnaît que la forme du village doit dépendre «de la nature et situation du terrain», mais que, «cependant la ronde ou la quarrée semble la plus commode, si le lieu la souffre» et que chacune des $20,30,40$ ou 50 habitations doit avoir une

11 Cette carte, par un nommé Harris, est intitulée A Mapp of the Improved Part of Pensilvania in America, Divided into Countyes, Townships and Lotts. Lettre de Michel Gaumond à l'auteur, 23 novembre 1990 et note de travail du 13 février 1986.

12 Instructions à Talon, 27 mars 1665, RAPQ (1930-31): 8; notes marginales de Talon, ibid., 16.

13 Talon à Colbert, 4 octobre 1665, ibid., 36.

14 Le même au même, 13 novembre 1666, ibid., 56. 
FIGURE 2

Extrait du plan de Gédéon de Catalogue, 1709

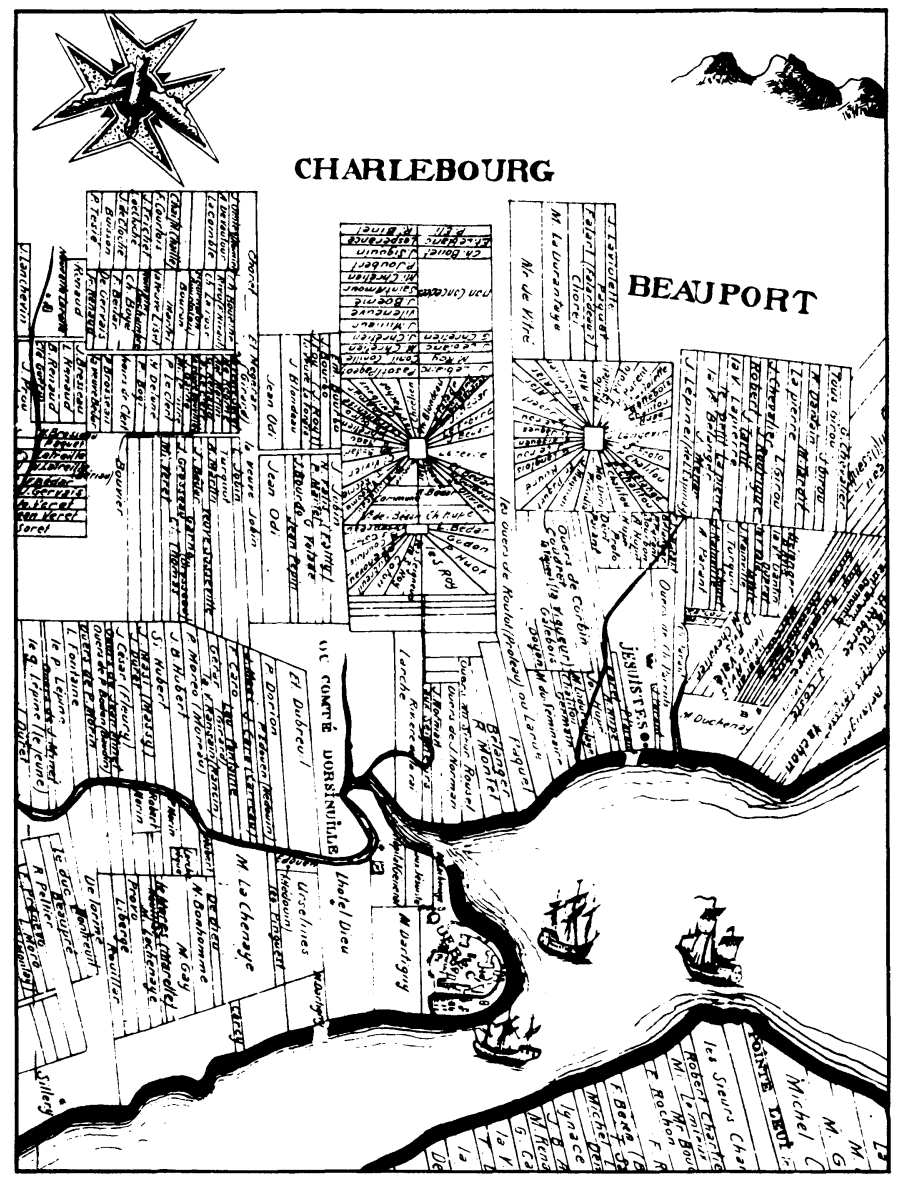

Au centre, nous apercevons le village-étoile de Charlesbourg; immédiatement au-dessous, la demi-étoile que forme le village Petite-Auvergne; à droite de Charlesbourg, le village-étoile de Bourg-Royal. 
superficie de 40 arpents, «avec un paître commun pour la garde commune des bestiaux» ${ }^{15}$.

Ce que l'on décide ici en 1667 est manifestement la formule déjà appliquée à Charlesbourg en février 1665 et répétée par les Jésuites en 1666 dans un autre village, appelé Petite-Auvergne ou Saint-Jérôme, tout juste au sud de Charlesbourg, avec ses terres en trapèze de 40 arpents chacune, mais où l'espace n'a pas permis cette fois de former une étoile complète, seulement une moitié, comme on le voit dans le plan de Catalogne (voir la figure 2). Si les autorités avaient voulu donner les sources de ce règlement de 1667, elles auraient pu citer Charlesbourg et Petite-Auvergne: elles n'y font aucune allusion.

Pourtant, Talon s'était déjà arrêté à une formule, puisqu'en novembre 1666, il annonce au ministre Colbert qu'il a entrepris trois villages, qui «sont desja bien avancés» ${ }^{16}$. Pour ce faire, il a «emprunté», comme il l'écrit, l'espace nécessaire dans la seigneurie de Notre-Dame-des-Anges, malgré l'opposition des Jésuites, espace qui sera d'abord soumis à la censive du roi, les travaux étant aux frais de l'État ${ }^{17}$. De ces trois villages, écrit Talon, deux sont destinés aux familles qu'on attend en 1667, un troisième est en voie de formation, grâce à de hauts-fonctionnaires civils et militaires ${ }^{18}$.

Talon parle de trois villages. Dans la partie est de Notre-Damedes-Anges, entre Charlesbourg et la limite de la seigneurie de Beauport, on reconnaît facilement Bourg-Royal, qu'identifie la carte de Villeneuve en 1688 et dont le dessin en étoile est reproduit dans le plan de Catalogne en 1709, comme on peut le voir sur la figure 2. Le deuxième est Bourg-la-Reine, au-dessus du précédent: nous pouvons le localiser par des actes notariés de 1670, mais le toponyme Bourgla-Reine n'apparaît pas dans cette carte de Villeneuve et il n'y a place, dans le plan de Catalogne, à côté du village en étoile de Charlesbourg, que pour un seul village en étoile, celui de Bourg-Royal. Et BourgTalon, où est-il? C'est un projet de village qui n'a pas été réalisé: lorsque les documents de cette époque font mention de Bourg-Talon, c'est pour l'assimiler à Bourg-Royal, comme c'est le cas d'actes de

15 Règlement du 24 janvier 1667, dans APC, colonies, $\mathrm{C}^{11} \mathrm{~A}$, vol. 2: 234-245; Ordonnances, commissions (éd. P.-G. Roy, 1924), I: 51-64; Édits, ordonnances royaux (éd. 1854), I: 29-34.

16 Talon à Colbert, 13 novembre 1666, RAPQ, 56.

17 Colbert à Talon, 5 avril 1667, ibid., 68; le même au même, 20 février 1668, ibid., 93; lettres d'érection du fief des Islets en baronnie, 14 mars 1671, dans APC, Ordres du roi, série B, vol. 1: 44-47; Inventaire des concessions en fief et seigneurie (éd. P.-G. Roy, 1927-28), II: 111. Cet espace «emprunté» sera en 1671 rattaché au fief des Islets, propriété personnelle de Talon.

18 Talon à Colbert, 13 novembre 1666, RAPQ, 56. 
concession de 1668 , de 1672 et de $1674^{19}$, ou comme dans la carte de 1688 sur laquelle Villeneuve inscrit Bourg Royal ou Bourg Talon ${ }^{20}$. Le village Bourg-Talon n'a pas existé comme entité distincte: il n'a été qu'un toponyme sans support réel. Les «trois villages» de Talon ne sont que deux: Bourg-Royal et Bourg-la-Reine.

Quant aux villages que Talon aurait érigés en étoile, il n'y en a qu'un seul, Bourg-Royal. À part Charlesbourg et Petite-Auvergne, celui-ci en demi-étoile ${ }^{21}$, qui sont l'œuvre des Jésuites, le plan de Catalogne ne reproduit qu'un seul village en étoile, celui de BourgRoyal: par quelques actes notariés, nous avons pu constater que les terres y ont un front d'un demi-arpent qui aboute à un trait-quarré, avec une profondeur qui va s'élargissant pour réunir les 40 arpents de superficie; d'où résulte la disposition en étoile.

Pour le Bourg-la-Reine, nous n'avons pu retracer que trois terres en forme de trapèze. Or le plan de Catalogne ne représente aucun village en étoile là où se situe Bourg-la-Reine, c'est-à-dire au-dessus de Bourg-Royal: les terres sont toutes de longues bandes étroites et rectangulaires; si la forme en étoile y a d'abord été tentée, on l'a tout de suite abandonnée: elle ne marque plus le paysage de 1709.

De Talon, donc, un seul village en étoile, Bourg-Royal, dit parfois Bourg-Talon, tracé par l'intendant d'après un plan que les Jésuites avaient appliqué à Charlesbourg six mois avant son arrivée dans la colonie. On a retenu que l'innovation en était due à Talon.

Ce qu'ont fait les premiers historiens de Charlesbourg. Charles Trudelle écrit en 1887 qu'à Charlesbourg les Jésuites ont imité le Bourg-Royal de Talon ${ }^{22}$, quand c'est l'inverse. Joseph Trudelle, en

19 Le toponyme Bourg-Talon apparaît dans divers actes pour désigner ce qui est BourgRoyal: un contrat de Talon avec Pierre Paradis, le 7 février 1668 (greffe Becquet), prévoit un chemin qui, sous le nom de «avenue du Bourg Talon» est dessiné par Villeneuve dans son plan de 1690 et partant de la terre de Paradis (dans Beauport). Des actes de concession, aussi dans Beauport, les 10 et 18 décembre 1668, à Denis Avisse, Robert Galien, Michel Accault, Jacques Savaria, Jacques Tardif, Pierre Sasseville, René Siret et Jean Langlois (greffe de Paul Vachon, aux Archives nationales), situent le bout de leurs terres au Bourg-Talon: il s'agit toujours de Bourg-Royal. Il en est de même en 1672 pour des actes de concession (encore dans Beauport) le 29 mars à Michel Baugis, le 22 mai à Pierre Sasseville, le 9 juillet à Étienne Dauphin et pour un acte de vente du seigneur Giffard à Toussaint Giroux le 24 mars 1672 (tous actes dans le même greffe de Paul Vachon). En 1674, la terre concédée à Jean Delespinay, le 27 novembre (greffe cité), se rend jusqu'au Bourg-Talon, c'est-à-dire Bourg-Royal.

20 Carte reproduite dans Trudel, Atlas historique du Canada français (Québec, Presses de l'Université Laval, 1961), 67.

21 Suite à des concessions faites dès 1666 , on a à Petite-Auvergne une vingtaine de terres de 40 arpents chacune qui s'aboutent en éventail au trait-quarré de 25 arpents (actes de concession dans le greffe de Paul Vachon, aux Archives nationales).

22 Charles Trudelle, Paroisse de Charlesbourg (Québec, Côté, 1887), 10-11. 
1896, est plus explicite: «Charlesbourg débuta sous l'œil de Talon. On peut dire que c'est la seule paroisse qui porte encore l'empreinte de l'idée créatrice du célèbre Intendant. Il en traça lui-même le plan, et en délimita les concessions. $\aleph^{23}$ Reine Malouin, historienne plus récente de Charlesbourg, voit dans ce système «une idée géniale de Jean Talon ${ }^{24}$.

D'autres historiens attribuent le même mérite à Talon. Ainsi, après avoir parlé des terres «triangulaires» et des «habitations construites aux sommets des triangles», Thomas Chapais ajoute: "Vous voyez la pensée de Talon imprimée sur ce sol fertile en caractères ineffaçables. $\aleph^{25}$ Gustave Lanctôt, sans dire un mot des Jésuites, décrit en 1964 le rôle de Talon dans la conception de ces villages, «dont les terres en triangle étaient orientées du centre, comme les rais d'une roue ${ }^{26}$. Selon André Vachon, qui lui non plus ne parle pas des Jésuites, Talon «adopta pour ses bourgades un plan tout à fait nouveau au Canada ${ }^{27}$. Un spécialiste du régime seigneurial, R. C. Harris, fait de même en $1966^{28}$, comme aussi encore en 1990 , le géographe Serge Courville $^{29}$. Mais battons aussi notre coulpe: sur la foi des historiens qui nous avaient précédé, nous avons nous-même attribué à Talon le village en étoile ${ }^{30}$...

C'est tout récemment, en 1987, dans Habitabec, journal où l'on ne s'attend guère à une information historique, que l'on apporte pour la première fois une rectification sur la paternité du village en étoile: un reportage y résume l'essentiel de la recherche (non encore publiée) de Michel Gaumond, qui attribue aux Jésuites ce qui leur est dû ${ }^{31}$. Grâce à Gaumond, R. C. Harris a pu se corriger et nous corriger tous, en écrivant dans l'Atlas historique du Canada: «Dans la seigneurie de Notre-Dame-des-Anges, au nord de Québec, les Jésuites distribuent les terres suivant un plan en forme d'étoile que maintient l'intendant

23 Joseph Trudelle, Charlesbourg (Québec, Faveur, 1896), vi.

24 Reine Malouin, Charlesbourg, 1660-1949 (Québec, La Liberté, 1972), 125, 127.

25 Thomas Chapais, Jean Talon (Québec, Demers, 1904), 161. Chapais parle de triangles: c'est l'aspect qu'ont les terres dans le plan de Catalogne, mais elles sont bien en forme de trapèze. Lanctôt et Malouin commettent la même erreur.

26 Gustave Lanctôt, Histoire du Canada (éd. 1964), II: 55.

27 André Vachon, «Talon», Dictionnaire biographique du Canada, I: 633.

28 R. C. Harris, The Seigneurial System in Early Canada (Québec, Presses de l'Université Laval, 1966), 172.

29 Serge Courville, Entre ville et campagne. L'essor du village dans les seigneuries $d u$ Bas-Canada (Québec, Presses de l'Université Laval, 1990), 15-18.

30 Marcel Trudel, Atlas de la Nouvelle-France (Québec et Toronto, Presses de l'Université Laval, 1973), 167.

31 Marcel Calfat, "La seule ville canadienne à posséder un "trait-carré"», Habitabec, 6 mars 1987. 
Talon lorsqu'il exproprie une partie de la seigneurie en $1666 .{ }^{32}$ Il restait à en publier une démonstration méthodique, ce que nous avons cru utile de faire.

En cette affaire, Talon a complètement passé sous silence, tout en le suivant d'une façon exacte, le plan en étoile de Charlesbourg. Il s'est ainsi assuré, devant le ministre Colbert et devant la postérité, le mérite d'une invention qui n'était pas la sienne. On doit ajouter que cela lui a été facilité de son vivant par le silence des Jésuites: nulle part, ni dans leurs Relations ni dans leur Journal (mais ce que nous en avons prend fin en 1668), il ne font état du système ingénieux qu'ils étaient les premiers à mettre en œuvre. C'est seulement quatre ans après la mort de Talon et redevenus les propriétaires de ce qu'il leur avait enlevé, que les Jésuites, dans un document privé de 1698, déclarent que ce sont eux qui «ont tiré les alignements» de Charlesbourg et que Talon n'a fait que suivre ${ }^{33}$.

32 Voir l'article de R. C. Harris dans Atlas historique du Canada, des origines à 1800 (publié en 1987 sous la direction de ce même Harris, aux Presses de l'Université de Montréal), vol. I: planche 52 .

33 Raisons que les Pères Jésuites ont eu de s'opposer à l' insinuation de la donation faite du comté d'Orsainville et ses dépendances à l'hospital général, document conservé aux archives de l'Hôpital-Général de Québec (communication de Michel Gaumond). 